\title{
Investigating the protective effect of tanshinone IIA against chondrocyte dedifferentiation: a combined molecular biology and network pharmacology approach
}

\author{
Yushen Zhang ${ }^{1}$, Liguo Sun ${ }^{1}$, Xincheng Liu ${ }^{1}$, Dongze Zhu ${ }^{1}$, Jingyi Dang ${ }^{1}$, Yingsen Xue ${ }^{2}$, Hongbin Fan ${ }^{1}$ \\ ${ }^{1}$ Department of Orthopedic Surgery, Xi-jing Hospital, Fourth Military Medical University, Xi'an, China; ${ }^{2}$ Department of Orthopedic Surgery, \\ Northwest Women and Children's Hospital, Xi'an, China \\ Contributions: (I) Conception and design: H Fan, Y Zhang; (II) Administrative support: L Sun, X Liu; (III) Provision of study materials or patients: \\ Y Zhang, L Sun, X Liu; (IV) Collection and assembly of data: D Zhu, J Dang, Y Xue; (V) Data analysis and interpretation: Y Zhang, H Fan; (VI) \\ Manuscript writing: All authors; (VII) Final approval of the manuscript: All authors. \\ Correspondence to: Hongbin Fan. Department of Orthopedic Surgery, Xi-jing Hospital, Fourth Military Medical University, Xi'an 710032, China. \\ Email: fanhongbin75@yahoo.com.
}

Background: Osteoarthritis (OA) is a common degenerative disease with multifactorial etiology. The dedifferentiation of chondrocytes can accelerate the progress of OA. Tanshinone IIA (TIIA) has been widely used to treat OA for many years and has proved to be effective in inhibiting chondrocyte dedifferentiation. Until now, the precise mechanism of TIIA's effect against dedifferentiation has not been well understood.

Methods: The targets of TIIA were explored from public databases using various methods. The related targets of OA were obtained from the GeneCards database and the Online Mendelian Inheritance in Man (OMIM) database. The potential targets and signaling pathways were determined using protein-protein interaction (PPI), Gene Ontology (GO), and the Kyoto Encyclopedia of Genes and Genomes (KEGG). Cell viability, proliferation, and metabolic activity were analyzed in vitro. The effects of TIIA on chondrocyte dedifferentiation were evaluated by assessing morphological changes, glycosaminoglycan (GAG) production, and messenger RNA (mRNA) levels of cartilage-related genes. After 48 hours of culture in medium with $100 \mu \mathrm{g} / \mathrm{mL}$ TIIA, chondrocytes/hydrogel spheres were implanted to repair cartilage defects in a rat model. The harvested specimens were examined with hematoxylin and eosin (H\&E) staining and immunohistochemistry to evaluate cartilage regeneration.

Results: The results showed that there were 28 genes potentially interacting in the TIIA-chondrocyte dedifferentiation network, and nine hub genes were identified. In vitro experiments showed an inhibitory effect of TIIA on chondrocyte dedifferentiation. The proliferation and viability of chondrocytes were promoted by TIIA at a concentration of $100-200 \mu \mathrm{g} / \mathrm{mL}$, but inhibited by TIIA at $400 \mu \mathrm{g} / \mathrm{mL}$. Furthermore, the histology results showed that chondrocyte/hydrogel spheres pre-treated with TIIA had better cartilage repair.

Conclusions: This study revealed a systematic network pharmacology approach and provided a basis for the future study of TIIA as an effective treatment for cartilage regeneration. Moreover, in vitro and in vivo results confirmed the protective effects of TIIA against chondrocyte dedifferentiation.

Keywords: Chondrocyte; tanshinone IIA (TIIA); dedifferentiation; network analysis; cartilage repair

Submitted May 17, 2020. Accepted for publication Oct 12, 2020.

doi: $10.21037 /$ atm-20-4023

View this article at: http://dx.doi.org/10.21037/atm-20-4023

$\wedge$ ORCID: 0000-0002-2955-6067. 


\section{Introduction}

Osteoarthritis (OA) is a chronic degenerative disease with multifactorial etiology. No disease-modifying therapy for OA has been developed to date (1-3). There are several risk factors for OA, including genetics, body mass index, and injury, which can also contribute to disease progression. However, the exact etiology is still unknown. A variety of treatment modalities are available for the management of OA. Non-pharmacological interventions include weight loss and physical therapy (4). Nevertheless, for effective disease control, a combination of non-pharmacological and pharmacological interventions is needed. Traditional drugs include acetaminophen (paracetamol), nonsteroidal anti-inflammatory drugs (NSAIDs), glucocorticoids, and intra-articular (IA) injection of hyaluronic acid (5). In the treatment of end-stage OA, joint replacement is one of the most commonly performed orthopedic procedures.

An increasing amount of evidence indicates that chondrocytes acquire a dedifferentiated phenotype during the process of $\mathrm{OA}(5)$, and their morphology usually changes from polygon into a spindle shape. The extracellular matrix (ECM) component is mainly characterized by decreased contents of glycosaminoglycan (GAG), collagen type II (Col II), and aggrecan (ACAN), and increased amounts of collagen type I (Col I), collagen type VI (Col VI), collagen type IX (Col IX), and collagen type X (Col X) (6). The validated markers of dedifferentiated chondrocytes are closely related to OA cartilage damage. These OA-linked deleterious factors can further accelerate or enhance the process of dedifferentiation (7). Given that chondrocytes can lose their specific phenotype in the process of OA, various methods have been developed in order to retain their phenotype.

Natural plant products have served as medicines for thousands of years. In particular, Traditional Chinese Medicine (TCM) has been widely used in clinical therapy. Increasing research suggests that its curative efficacy and small number of side effects might make it a key strategy for the treatment of OA $(8,9)$. Salvia miltiorrhiza Bunge, commonly known as Danshen (Chinese Pinyin name), has been widely used for the prevention and treatment of OA (10), coronary artery disease, and liver fibrosis in China for thousands of years. Tanshinone IIA (TIIA, C19H18O3, PubChem CID:164676) is a class of abietane diterpene compounds isolated from Salvia miltiorrhiza Bunge, which have widespread pharmacologic effects including antiinflammatory effects (11), anti-oxidant capacity (12), anticancer properties (13), and anti-atherosclerotic effects (14).
Despite these findings, the molecular mechanisms underlying the protective effects of TIIA on chondrocytes are not fully understood. Furthermore, identifying a clear mechanism with routine pharmacological evaluation is challenging.

With the rapid development of bioinformatics and systems biology, the network pharmacology approach has emerged as a powerful tool for investigating the targets and therapeutic effects of drug interventions (15). A recent network pharmacology approach demonstrated that TIIA could alleviate liver fibrosis (16). We therefore hypothesized that bioinformatics analysis and pharmacological network analysis could be used to identify potential target genes and pathways involved in chondrocyte dedifferentiation, as well as to determine the protective effects of TIIA.

In order to address this hypothesis, the current study was designed to: (I) screen action targets related to TIIA and chondrocyte dedifferentiation; (II) construct an interaction network for putative targets of TIIA and dedifferentiation; (III) predict the possible mechanism of TIIA on chondrocyte dedifferentiation; (IV) detect the cell viability, proliferation, and transcription level of cartilage-related genes including SOX6, Col I, Col II, and Col X in dedifferentiated chondrocytes; (V) repair cartilage defects with chondrocyte/ hydrogel beads after treatment with TIIA. A flowchart of the study design is illustrated in Figure 1.

We present the following article in accordance with the ARRIVE reporting checklist (available at http://dx.doi. org/10.21037/atm-20-4023).

\section{Methods}

\section{Data preparation}

The structure of TIIA (Figure $2 A$ ) was obtained from PubChem (https://pubchem.ncbi.nlm.nih.gov/). According to this structure, several databases including Pharmmapper (http://www.lilab-ecust.cn/pharmmapper/) (17), Swiss Target Prediction (http:// www. swisstargetprediction. ch/) (18), and Drug bank (https://www.drugbank.ca/) (19) were used to obtain as many potential targets as possible. Thereafter, pharmacokinetic properties including absorption, distribution, metabolism, and excretion (ADME) were determined as the key contributors affecting bioactivities. TIIA was searched in databases including the TCM systems pharmacology database and analysis platform (TCMSP, http://tcmspw.com/tcmsp.php) (20), Bioinformatics Analysis Tool for Molecular mechANism of TCM (BATMAN, http:// bionet.ncpsb.org/batman-tcm/index.php/Home/Index/ 


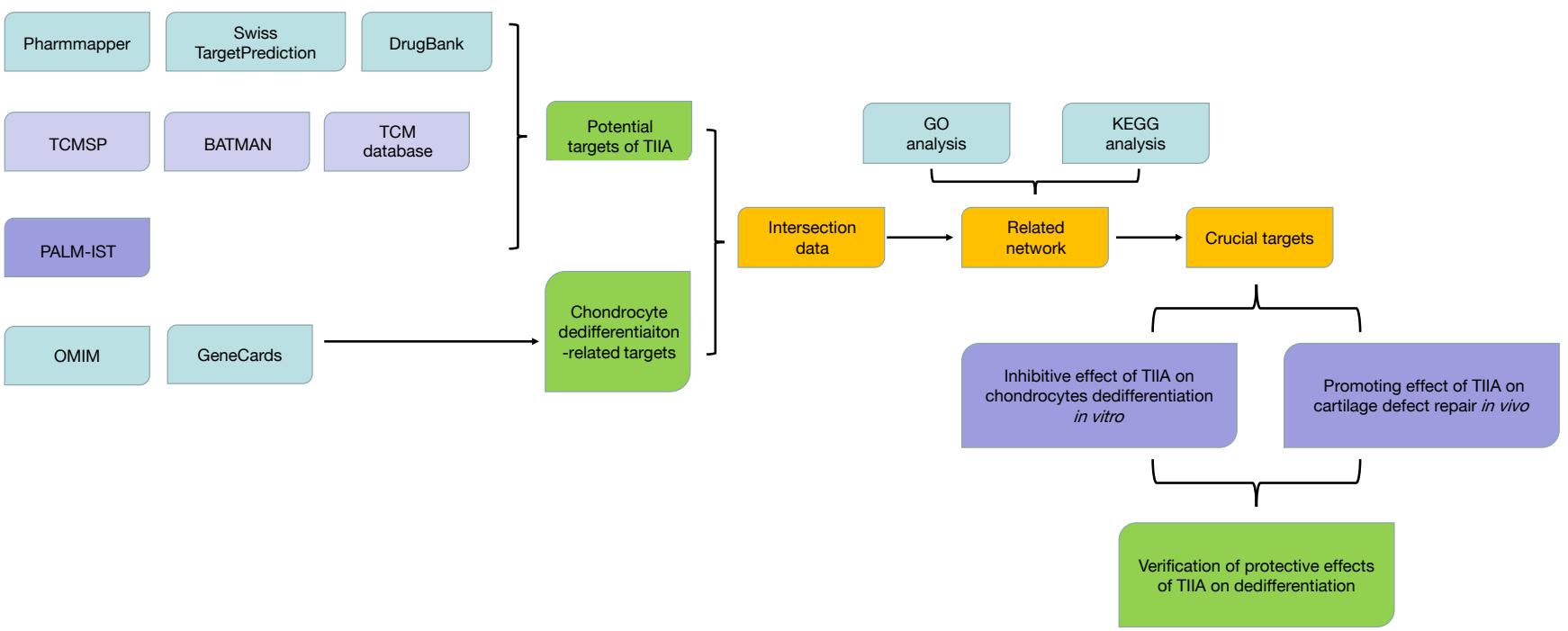

Figure 1 Flow diagram of the study design. The overall design is based on the methods of network pharmacology and experimental verification. GO, Gene Ontology; KEGG, Kyoto Encyclopedia of Genes and Genomes; TIIA, tanshinone IIA.
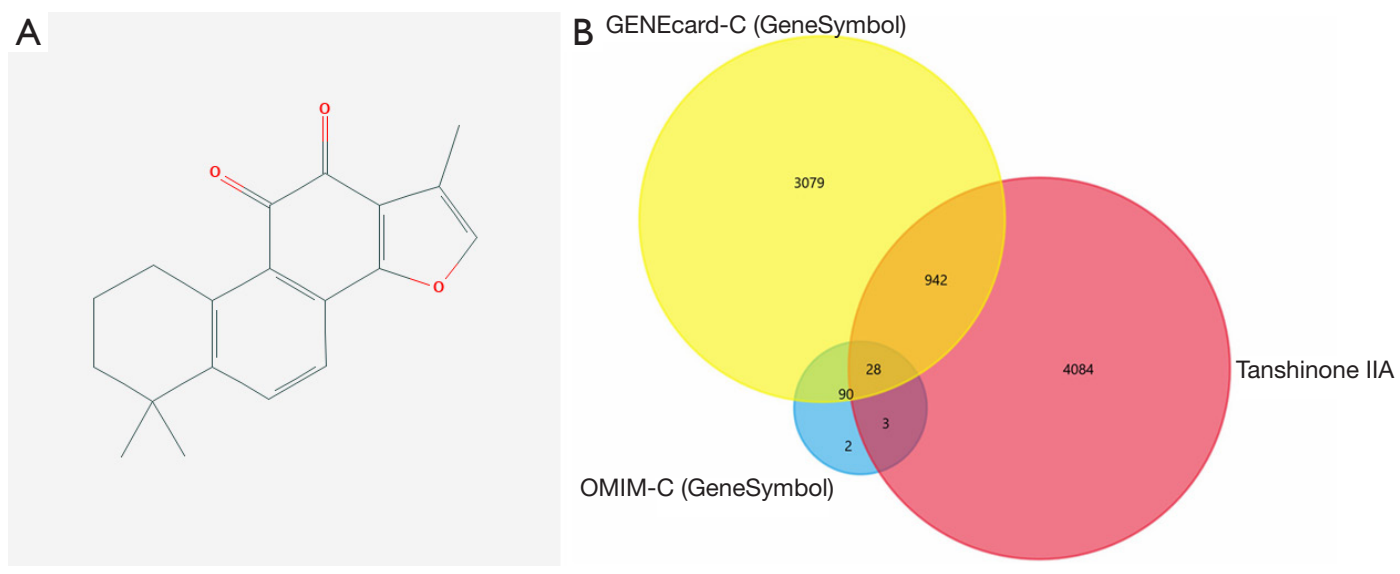

Figure 2 Data preparation process. (A) The two-dimensional structure of TIIA; (B) Venn diagram of candidate targets exhibiting the interaction of TIIA and chondrocyte dedifferentiation. TIIA, tanshinone IIA.

index) (21), and the TCM Database (http://tcm.cmu.edu. $\mathrm{tw} /$ ) (22), and screened with oral bioavailability (OB) $\geq 30 \%$ and drug-likeness (DL) $\geq 0.18$ as the included criteria. Next, the potential targets of TIIA were collected by text mining with the Pathway Assembly from Literature Mining-an Information Search Tool (PALM-IST, http://www.hpppi. iicb.res.in/ctm/index.html) (23), which is a computational platform for users to explore biomedical literature resources (PubMed) using multiple keywords.

The chondrocyte dedifferentiation-related protein targets were screened using two existing resources, GeneCards (https://www.genecards.org/) and the Online Mendelian
Inheritance in Man database (OMIM, https://omim.org/), and the keywords "chondrocyte" and "dedifferentiation". Irrelevant results were removed manually and all results were limited to Homo sapiens.

All target proteins were standardized according to the Uniprot website (https://www.uniprot.org/) and the intersection data were taken (Figure 2B).

\section{Related network construction and analysis}

An interactive network was constructed with the putative targets of TIIA and cartilage dedifferentiation data. Protein 
interaction was studied using STRING (https://string-db. org/cgi/input.pl) and Cytoscape.

Next, related targets were imported to visualize the interaction network. For modularity analysis, two Cytoscape Network Analyzer plugins, molecular complex detection (MCODE) and CytoHubba, were used. MCODE was based on vertex weighting by local neighborhood density and outward traversal from a locally dense seed protein to isolate the dense regions according to given parameters. The algorithm had the advantage of permitting a directed mode that recognized all possible molecular complexes of particular protein networks. Meanwhile, Cytohubba ranked the nodes according to the attributes in the network, which provided 11 topological analysis methods. Densely connected regions in large protein-protein interaction (PPI) networks that might represent molecular complexes were detected.

To further understand the signaling pathways and functions of these genes, the targets of genes and hub genes were screened in functional enrichment analysis using DAVID (https://david.ncifcrf.gov/) and Metascape (http:// metascape.org/gp/index.html\#/main/step1) to annotate the Kyoto Encyclopedia of Genes and Genomes (KEGG) pathway and Gene Ontology (GO) terms. GO terms and KEGG pathways with $\mathrm{P}$ values $<0.05$ were considered statistically significant. The files could also be imported directly into the FunRich (24) tool for further analysis; this was carried out for additional visualization of the results and provided an intuitive impression of gene proportions.

\section{Verification of the inbibitory effects of TILA on chondrocyte dedifferentiation}

\section{Animals}

All Sprague Dawley (SD) rats were purchased from the Experimental Animal Center of the Fourth Military Medical University (Xi'an, China). For in vitro assays, chondrocytes were isolated from the knee joint cartilage of rats (weight: 40-50 g; 2 weeks old). For the in vivo experiments in this study, female SD rats (weight: 180-200 g; 6 weeks old) were used. All procedures complied with the Animals (Scientific Procedures) Act 1986. All procedures involving animals were approved by the Animal Care and Use Committee of the Fourth Military Medical University (permission number: FMMU20180712), and were implemented complying with the Guidelines for the Care and Use of Laboratory Animals. All rats were maintained at $25 \pm 2{ }^{\circ} \mathrm{C}$ and $40-60 \%$ relative humidity on a 12 -hour light/dark cycle with free access to food and water.

\section{Cell culture and treatment}

Chondrocytes were harvested from the knee joints of SD rats using the collagenase digestion method. Cells were resuspended in Dulbecco's Modified Eagle Medium (DMEM, Gibco, USA) supplemented with $10 \%$ fetal bovine serum (FBS, Gibco) and cultured at $37{ }^{\circ} \mathrm{C}$ with $5 \% \mathrm{CO}_{2}$. When cells reached $70-80 \%$ confluency, adherent cells were trypsinized, harvested, and further expanded to passage 4 (P4). Chondrocytes (P4) were then cultured in basal medium with various concentration of TIIA for further experiments as follows: (I) group A: cultured in basal medium; (II) group B: cultured in basal medium with TIIA $(100 \mu \mathrm{g} / \mathrm{mL})$; (III) group C: cultured in basal medium with TIIA $(200 \mu \mathrm{g} / \mathrm{mL})$; (IV) group D: cultured in basal medium with TIIA $(400 \mu \mathrm{g} / \mathrm{mL})$. The basal medium contained high glucose DMEM, 10\% FBS, and $1 \%$ penicillin/streptomycin (P/S, $100 \mathrm{U} / \mathrm{mL})$. All reagents were of analytical grade and the other non-specified reagents were form Solarbio (Solarbio, China).

\section{Cell viability}

Cell counting kit-8 (CCK-8) assay (Dojindo, Japan) was used to determine the viability of cells. Cells (P4) from groups A, B, C, and D were seeded in each well of a 96 -well plate with 6 repeats $\left(2 \times 10^{3} /\right.$ well). CCK- 8 solution $(20 \mu \mathrm{L})$ was added to each well after cells were incubated for 24, 48, or 72 hours, and further incubated for 4 hours at $37{ }^{\circ} \mathrm{C}$. The optical density (OD) values were recorded at a wavelength of $540 \mathrm{~nm}$. The experiments were performed 4 times independently.

\section{Examination of cell morphology and Alcian blue staining}

Cells from groups A, B, C, and D were cultured in 6-well plates for 72 hours. Morphological changes in the cells were then observed under an inverted microscope (Olympus, Japan).

Proteoglycans were evaluated with Alcian blue staining. Briefly, each well was hydrated through a graded ethanol series and stained with $1 \%(\mathrm{w} / \mathrm{v})$ Alcian blue (Sigma, USA) solution in $3 \%(\mathrm{w} / \mathrm{v})$ glacial acetic acid, $\mathrm{pH} 4.2$, for 30 minutes, followed by washing with tap water for 10 minutes. The results were analyzed using Image J software to semi-quantify the average light density [integral OD (IOD)/area] values. 
Table 1 Sequences of RT-PCR primers

\begin{tabular}{ll}
\hline Gene & Forward/reverse \\
\hline Col1A1 & F: 5'-GGGATTCCCTGGACCTAAAG-3' \\
Rol2A1 & F: 5'-GGAACACCTCGCTCTCCAG-3' \\
& R: 5'-TCCCAGTGTCACAGACACAGAT-3' \\
Col10A1 & F: 5'-TCCCAGAACCCAGAATCCATC-3' \\
& R: 5'-GGTTGTGGGCCTTTATGCC-3' \\
SOX6 & F: 5'- TCAAAGGCAATTTACCAGTGATT-3' \\
& R: 5'- GGCTTGCTTGGAAGACATTC-3' \\
GAPDH & F: 5'-CTCTGCTCCTCCTGTTCGAC-3' \\
& R: 5'-ACGACCAAATCCGTTGACTC-3'
\end{tabular}

RT-PCR, real-time polymerase chain reaction; Col, collagen; SOX6, SRY-box transcription factor 6; GAPDH, glyceraldehyde 3-phosphate dehydrogenase.

\section{Real-time quantitative polymerase chain reaction (RT- $q P C R)$}

A RT-qPCR analysis was carried out. The sequences of specific primer sets were listed in Table 1. According to the manufacturer's instructions, total RNA was extracted from the chondrocytes of each group using TRIzol reagent (Sigma, USA). RNA was reverse transcribed into cDNA, then PCR-amplified using a TM One-Step RT-PCR kit with SYBR green reagent (Applied Biosystems, USA). The transcription level of genes was determined using the comparative Ct method.

\section{Repair of cartilage defects}

\section{Preoperative preparation}

SD rats (weight: 180-200 g; 6 weeks old) were purchased from Experimental Animal Center of the Fourth Military Medical University (Xi'an, China). Chondrocytes from the SD rats were applied to hydrogel spheres $(2 \%$ alginate + $1 \%$ sodium hyaluronate) at a cell density of $4 \times 10^{5}$ cells $/ \mathrm{mL}$ (Figure 3A,B). The chondrocytes/spheres were cultured for 48 hours in basal medium with or without $100 \mu \mathrm{g} / \mathrm{mL}$ TIIA. According to our previous study (25), the concentration of $100 \mu \mathrm{g} / \mathrm{mL}$ is effective for inhibiting chondrocyte dedifferentiation.

\section{Cartilage defect repair}

A full-thickness defect (thickness: $1.2-1.5 \mathrm{~mm}$; diameter:
$0.8 \mathrm{~mm}$ ) was created through the articular cartilage in the aforementioned rats using a drill bit with a diameter of $0.8 \mathrm{~mm}$ (Figure 3C). Eighteen female SD rats were randomly assigned into three groups. The cell-loaded spheres with TIIA pre-treatment were implanted to fill chondral defects in group $A(n=6)$. In the control group, cell-loaded spheres without TIIA pre-treatment were implanted (group B, $\mathrm{n}=6$ ). In the blank control group (group $\mathrm{C}, \mathrm{n}=6$ ), the defects were untreated. After surgery, all rats were allowed to move freely without plaster immobilization. All animals were sacrificed for subsequent analysis at 21 days postoperatively.

\section{Histology analysis}

For histological analysis, samples were harvested on day 21 postoperatively. The distal part of the femur was removed and fixed in $4 \%$ paraformaldehyde, decalcified in $10 \%$ ethylene diamine tetraacetic acid (EDTA), and embedded in paraffin. The samples were sectioned at a thickness of $10 \mu \mathrm{m}$ and stained with hematoxylin and eosin (H\&E), Safranin O/Fast Green, and Alcian blue. For immunohistochemical staining, the slides were incubated with primary antibodies for Col I, Col II, Col X, and SOX6 (Abcam, UK) at $4{ }^{\circ} \mathrm{C}$ overnight. A secondary antibody was added and incubated for 30 minutes. The sections were then incubated in horseradish enzyme-labeled chain avidin solution for 30 minutes at room temperature. The color was developed using a diaminobenzidine (DAB) substrate kit (Bioss Biotechnology, China). The results were evaluated by three individuals, who were blinded to the treatments.

\section{Statistical analysis}

Graphs were drawn using GraphPad Prism software and statistical analyses were performed using GraphPad Prism and SPSS. Comparisons between groups were analyzed using an unpaired Student's $t$-test. Multiple group comparisons were performed using one-way analysis of variance (ANOVA) and repeated-measures ANOVA. $\mathrm{P}<0.05$ was considered statistically significant.

\section{Results}

\section{Screening out putative targets for TIIA}

After the elimination of duplicates, 5,020 potential target genes of TIIA were identified in all databases. Additionally, 3,784 and 123 genes related to chondrocyte 

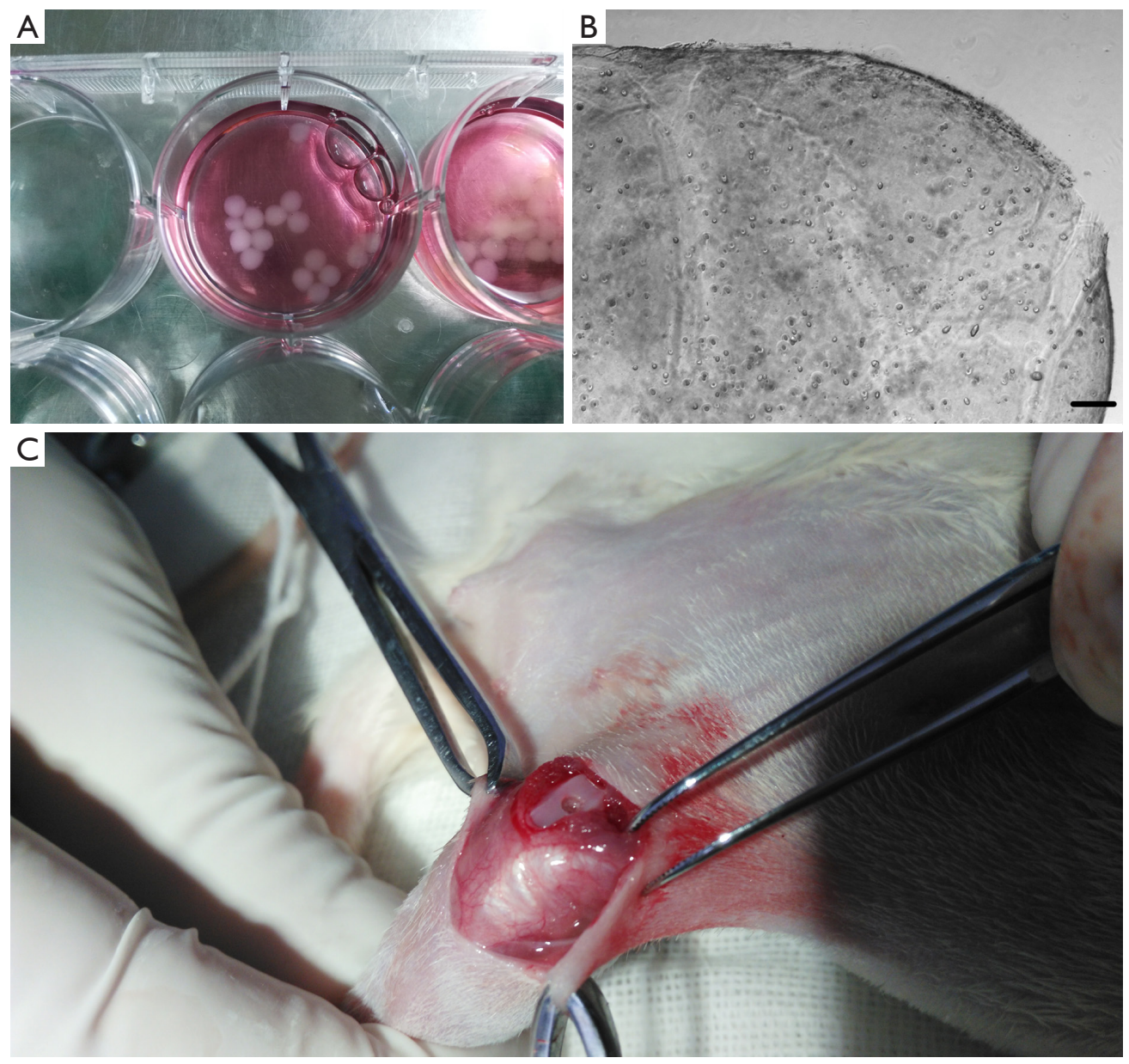

Figure 3 Hydrogel microspheres filling the cartilage defect in rat model. (A) Alg-HA hydrogel spheres in TIIA medium; (B) cells were observed under a light microscope $(50 \times$ magnification, scale bar $=50 \mu \mathrm{m})$; (C) intraoperative construction modeling of cartilage defects. AlgHA, alginate-sodium hyaluronate; TIIA, tanshinone IIA.

dedifferentiation were retrieved from the GeneCards and OMIM databases, respectively. From all the available resources, 28 overlapping putative protein targets were recognized from the two categories of targets described above (Figure $4 A$ ).

\section{Compound-target network analysis}

To elucidate the relationship between TIIA targets and chondrocyte dedifferentiation-related targets, the related network was constructed. By combining the results of STRING and Cytoscape, the PPI network comprised 33 nodes and 61 edges in total. In addition, seven hub genes including COL2A1, COL10A1, ADAMTS5, MMP13, $C O M P, G D F 5$, and $S O X 6$ represented the crucial targets based on degree and betweenness centrality (Figure $4 B$ ), and were ranked by color according to the score of MCODE and CytoHubba.

\section{Enrichment analysis of candidate targets for TIIA against dedifferentiation}

The enrichment analysis of the 28 putative targets was conducted using Metascape, which is a web-based portal designed to provide a comprehensive gene list annotation and analysis resource. As shown in Figure 4C, GO analysis and KEGG pathway enrichment analysis were performed to clarify the characteristics of TIIA-related targets. The major targets were categorized into various functional modules including connective tissue development, ECM 
A

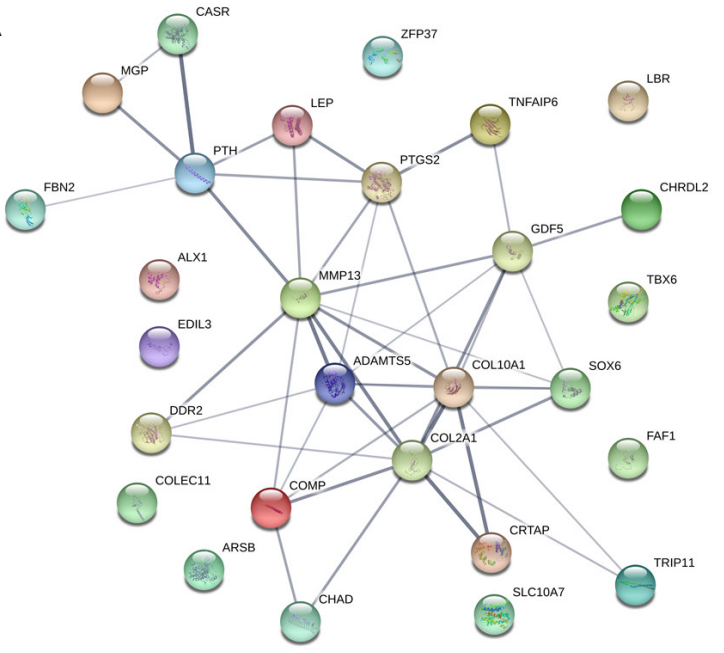

C

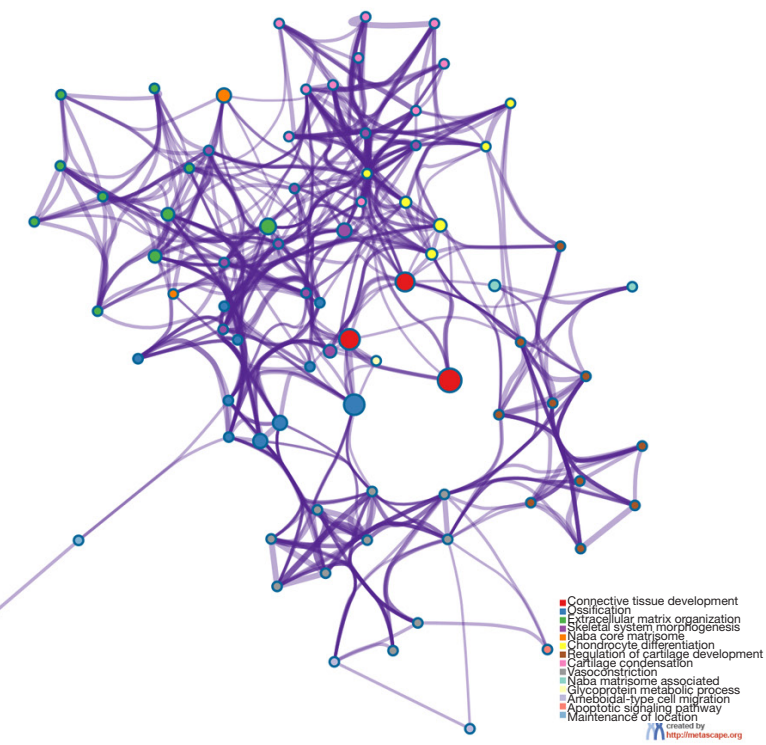

B
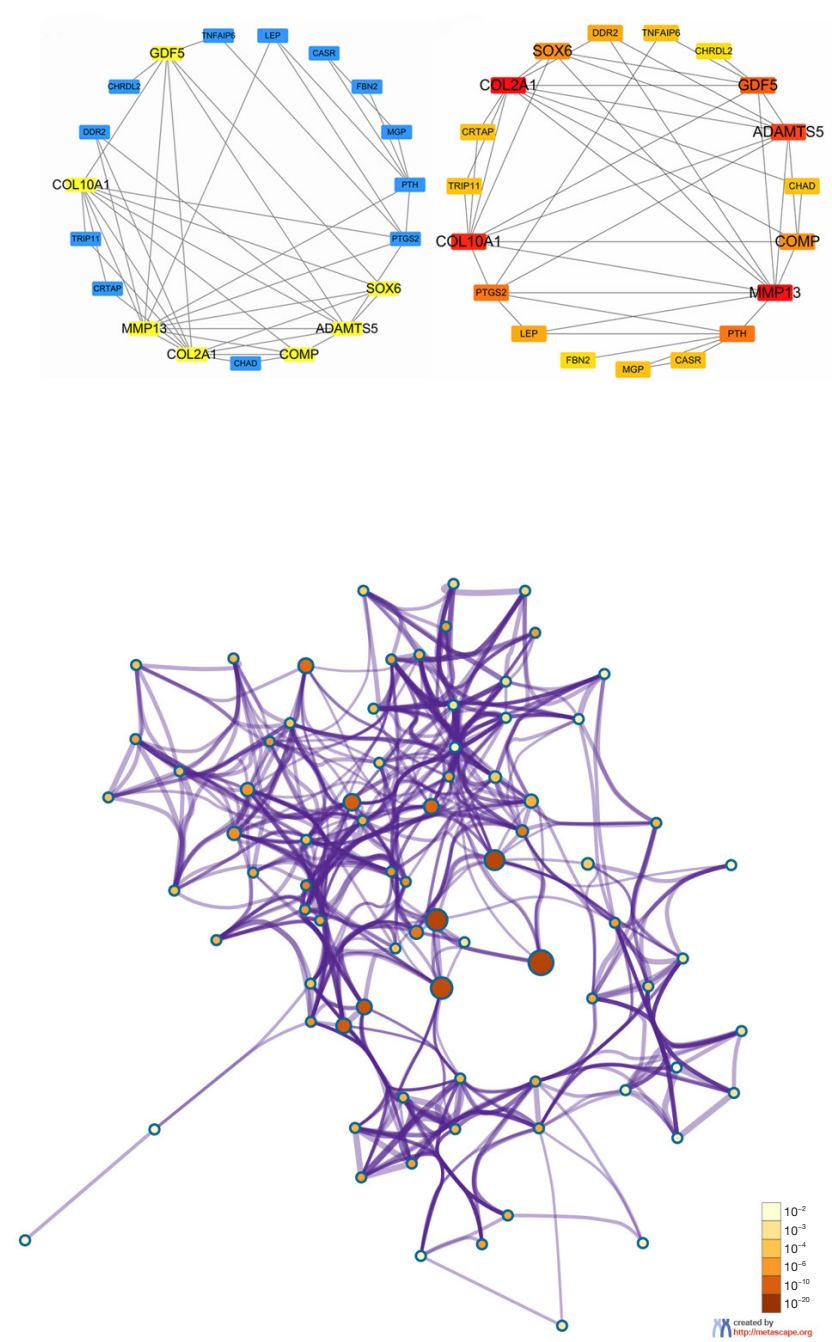

Figure 4 Results of network analysis. (A) PPI networks of the 28 genes of the intersection data; (B) hub gene networks. Left: hub genes selected by MCODE; right: hub genes ranked for color by CytoHubba; (C) the network of enriched terms of 28 putative targets. Left: colored by cluster ID, where nodes sharing the same cluster ID are typically close to each other; right: colored by $\mathrm{P}$ value, where terms containing more genes tend to have a more significant $\mathrm{P}$ value. PPI, protein-protein interaction.

organization, skeletal system development, ossification, chondrocyte differentiation, and regulation of cartilage development. These findings suggested that various biological processes such as gene transcription and expression, chondrocyte development, intercellular contacts, and ECM remodeling were involved in the multiple synergistic effects of TIIA against chondrocyte dedifferentiation.

\section{Inbibitory effect of TIIA on chondrocyte dedifferentiation in vitro}

The CCK- 8 assay indicated that the proliferation of chondrocytes in groups $\mathrm{A}, \mathrm{B}$, and $\mathrm{C}$ increased rapidly at the early stage of culture. The OD values for groups A, B, and C were $1.41 \pm 0.11,1.52 \pm 0.19$, and $1.64 \pm 0.17$, respectively, at 24 hours, and $1.68 \pm 0.13,2.63 \pm 0.16$, and $2.05 \pm 0.09$, 

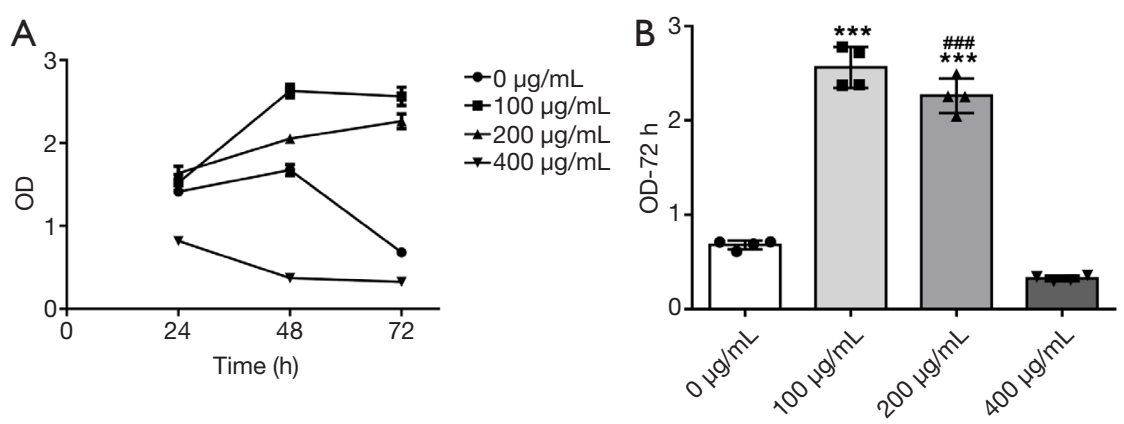

Figure 5 CCK-8 assay for cellular viability. (A) CCK-8 OD values of cartilage cells in each group at 0, 24, 48, and 72 hours; (B) bar graph of cartilage cell proliferation compared to control cells measured using the CCK-8 assay at 72 hours $\left(* * *, \mathrm{P}<0.001\right.$ vs. $0 \mu g$; ${ }^{\# \#}, \mathrm{P}<0.001 v s$. $400 \mu \mathrm{g}, \mathrm{n}=4)$. CCK-8, cell counting kit-8; OD, optical density.
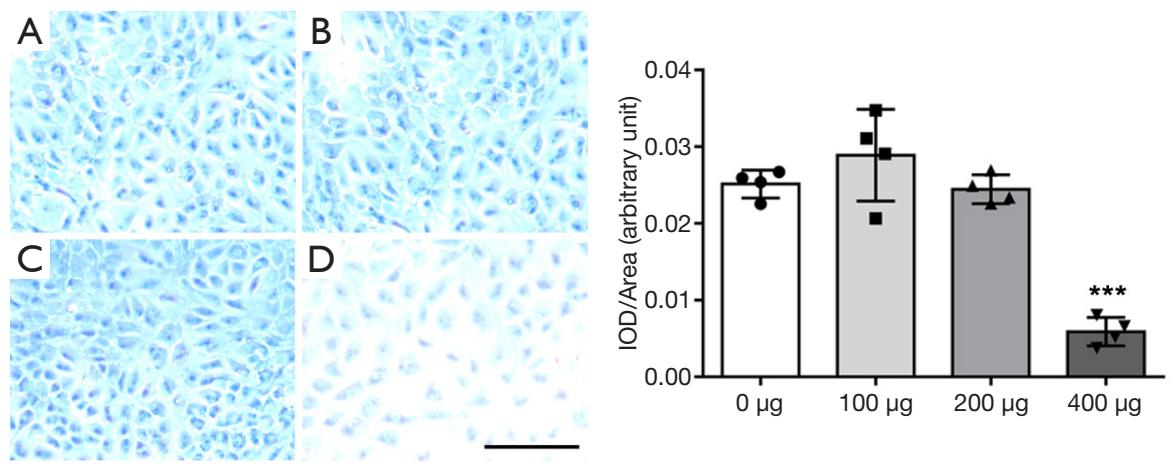

Figure 6 Alcian blue staining of cartilage cells in each group. Left: (A) control group without TIIA (0 $\mu \mathrm{g} / \mathrm{mL})$; (B) TIIA (100 $\mu \mathrm{g} / \mathrm{mL})$; (C) TIIA $(200 \mu \mathrm{g} / \mathrm{mL})$; (D) TIIA $(0 \mu \mathrm{g} / \mathrm{mL})(50 \times$ magnification, scale bar $=50 \mu \mathrm{m})$; right: the intensity of the blue area was analyzed with IOD/ area $(* * *, \mathrm{P}<0.001$ vs. $0 \mu \mathrm{g}, \mathrm{n}=4)$. TIIA, tanshinone IIA; IOD, integral optical density.

respectively, at 48 hours after cell seeding. Thereafter, the curve of group $\mathrm{C}$ increased steadily and reached a peak at 72 hours with a value of $2.26 \pm 0.18$. Contrastingly, the curves of groups A and B decreased, with values of $0.68 \pm 0.05$ and $2.56 \pm 0.22$ at 72 hours, respectively. The curve of group $\mathrm{D}$ initially decreased before reaching a plateau at 72 hours with a value of $0.33 \pm 0.03$. The values of groups $B$ and $C$ at 72 hours were significantly higher than those of groups A and $\mathrm{D}(\mathrm{P}<0.05)$ (Figure 5).

In groups $\mathrm{B}$ and $\mathrm{C}$, dark staining of Alcian blue indicated that more GAG was produced at the concentration of $100-200 \mu \mathrm{g} / \mathrm{mL}$ TIIA. In contrast, in group D, Alcian blue staining was light with the concentration of $400 \mu \mathrm{g} / \mathrm{mL}$ TIIA $(\mathrm{P}<0.05)$ (Figure 6).

As previously reported, the hub genes were ranked by color. RT-qPCR analysis was used to examine the mRNA levels of chondrogenic genes including COL1A1, COL2A1, COL10A1, and SOX6. Compared with those in group A, the mRNA levels of Col II and SOX6 were significantly up-regulated in groups B and C. On the other hand, the transcription levels of $\mathrm{Col} I$ and $\mathrm{Col} X$ genes were dramatically down-regulated. However, in group D, the expression levels of all chondrogenic markers were significantly lower (Figure 7). These results indicated that the dedifferentiation of chondrocytes was affected by TIIA at various concentrations.

\section{Effect of TILA on cartilage defect repair}

Figure 8 shows the macroscopic appearance of chondral defect repair at 21 days postoperatively. In group A, the implant was fused with host tissue and the interface had almost disappeared. The surface of the defects was more intact and smoother than that in group C. Alcian blue staining showed that the GAG content in group A was close to that in normal tissues. The chondrocytes in the repair 

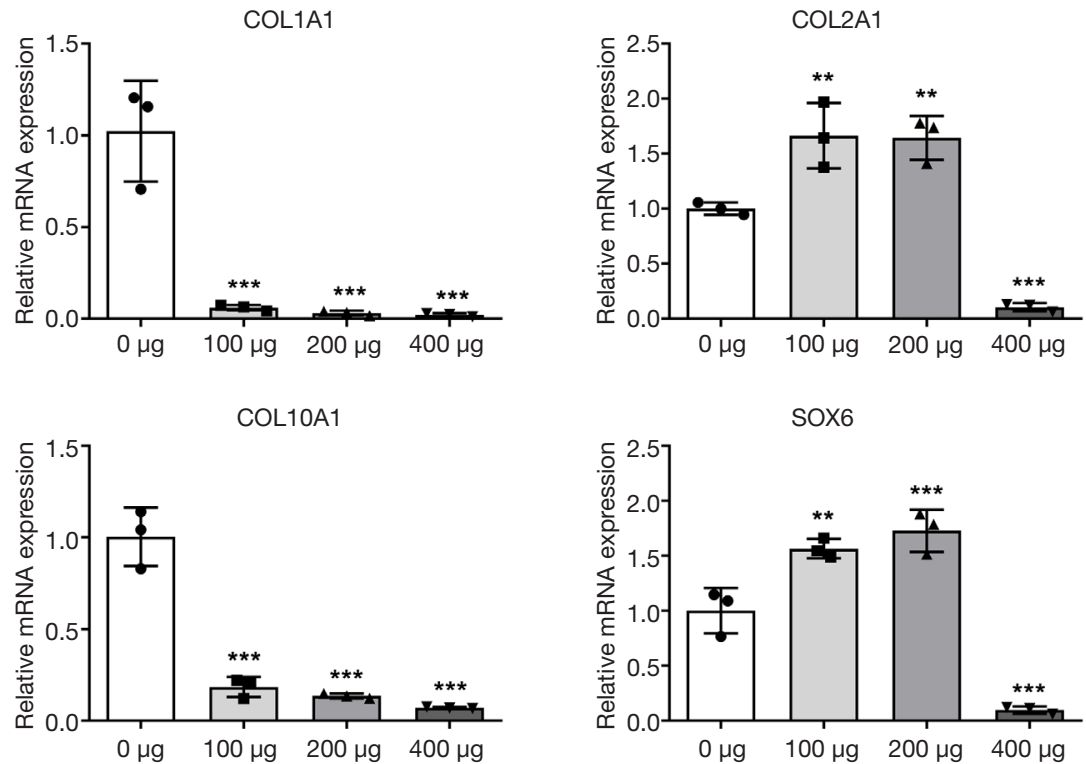

Figure 7 Results of real-time quantitative polymerase chain reaction for each cell treatment group $\left({ }^{* *}, \mathrm{P}<0.01,{ }^{* * *}, \mathrm{P}<0.001 v s .0 \mu g, \mathrm{n}=3\right)$.
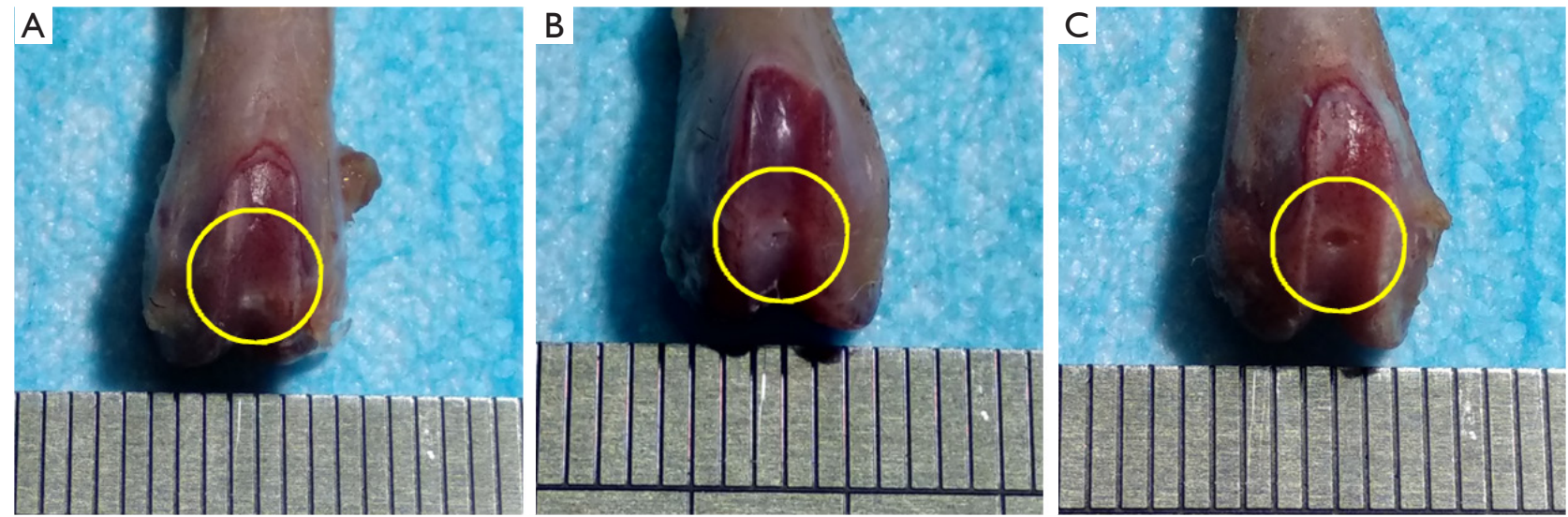

Figure 8 Knee joint specimen appearance in each treatment group. The area shown by the yellow circle represents the cartilage repair structure. (A) Group A, alginate + TIIA; (B) group B, Alginate; (C) group C, untreated. TIIA, tanshinone IIA.

region were observed to have a regular distribution and nearly normal morphology. Compared with group $\mathrm{C}$, the repair region showed good subchondral bone reconstruction and hyaline-like cartilage formation, with a high amount of Col II and a lower amount of Col I and X (Figure 9).

In contrast, in group $\mathrm{B}$, the subchondral bone was repaired incompletely, with less hyaline-like cartilage formation. The defect region was mostly filled with loose fibrotic tissue, and the surface was not smooth. In group C, a large blue-stained area was observed, which lacked normal cartilaginous structure. Higher levels of Col I and Col
$\mathrm{X}$, and a lower level of Col II, were also observed. These histological features indicated that pre-treatment with TIIA was beneficial for cartilage damage repair.

\section{Discussion}

$\mathrm{OA}$ is a pathological condition that is characterized by progressive cartilage degradation (5). During OA progression, dedifferentiated chondrocytes lose their chondrogenic characteristics and display a fibroblast-like morphology (26). In monolayer culture, the expanded 

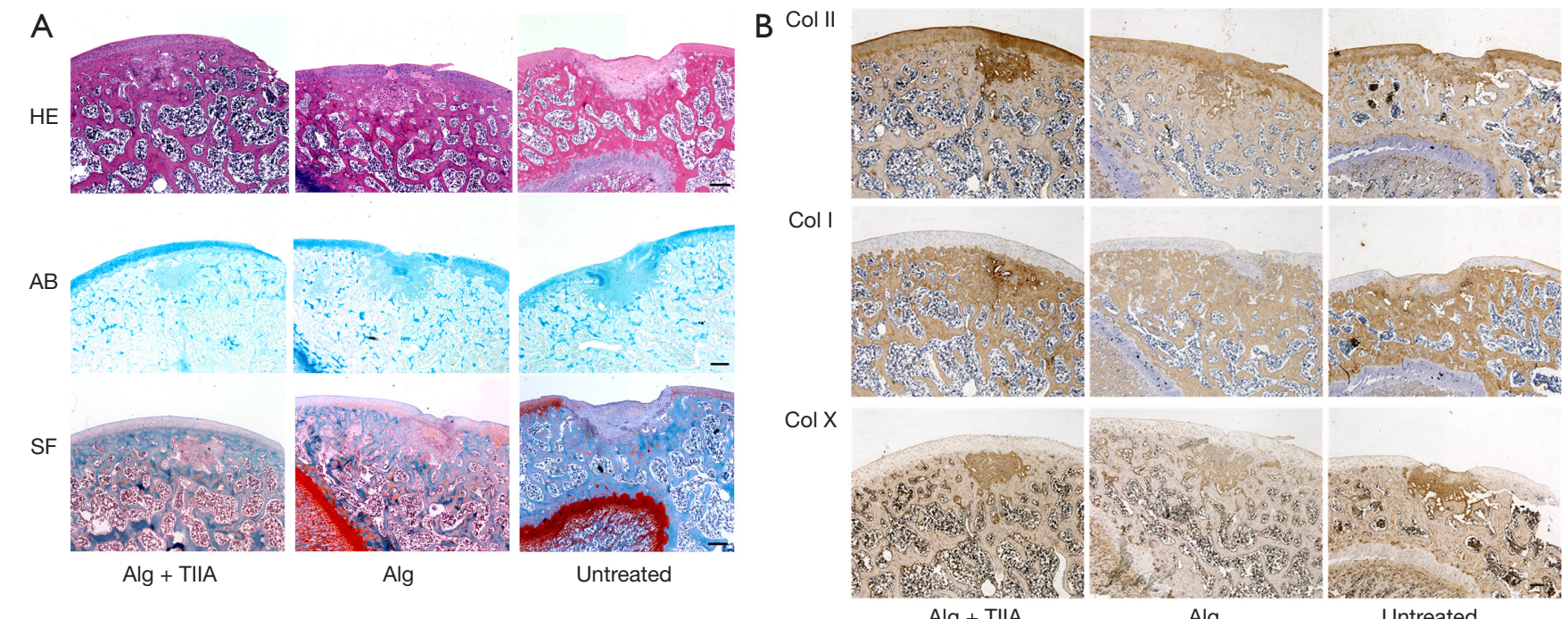

Alg + TIIA
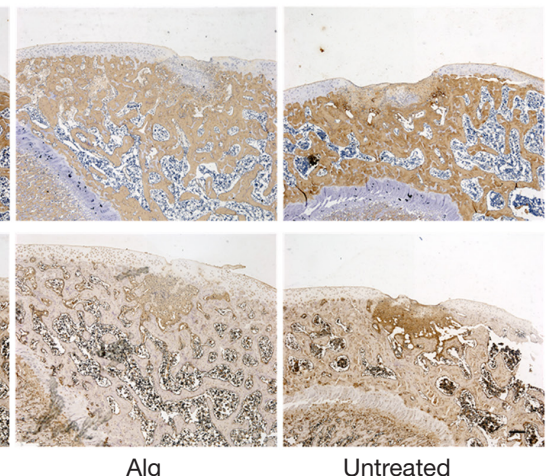

Figure 9 Histological staining of the knee joint in each treatment group $(50 \times$ magnification, scale bar $=50 \mu \mathrm{m})$. (A) Hematoxylin and eosin staining (HE); Alcian blue staining (AB); Safranin O/Fast Green staining (SF); (B) immunohistochemical staining in each group.

chondrocytes dedifferentiate more easily, losing their chondrogenic characteristics $(27,28)$. Therefore, differentiated chondrocytes in monolayer culture were used in this study to mimic dedifferentiated chondrocytes in OA. Common reasons for the development of OA include focal lesions, traumas, or articular overloading (28). Due to intrinsic avascularity and low cell population, damaged cartilage does not heal spontaneously. Therefore, the key issue facing pharmacological therapy for $\mathrm{OA}$ is to keep the chondrogenic phenotype of chondrocytes intact.

Using network pharmacology (29), the complexity of compounds and multiple targets of TCM can be better understood (30). Salvia miltiorrhiza Bunge (Danshen) has been clinically used in China for centuries to prevent and treat OA. Recently, it has been reported to prevent cartilage degeneration via its antioxidant capacity (31). The active components of Salvia miltiorrhiza Bunge, which include salvianolic acid, tanshinone, and isotanshinone, demonstrate oxidative resistance, free radical scavenging, and improvement of microcirculatory disturbance. TIIA, an important TCM monomer (32), has been used to treat coronary heart disease (33), stable angina (34), and liver fibrosis (35). A number of studies have indicated that TIIA has therapeutic effects for OA $(36,37)$. Our previous study also showed that TIIA played diverse roles in the dedifferentiation of chondrocytes, which were concentration-dependent (25). However, the therapeutic effects of TIIA on OA and their underlying mechanisms still need to be further explored.

In this paper, only 28 targets including seven hub genes were screened and found to participate in the interaction of cartilage dedifferentiation and TIIA. The results suggested that the protective effects of TIIA can facilitate connective tissue development, ECM organization, skeletal system development, cartilage development, and chondrocyte differentiation, to regulate the occurrence and development of chondrocyte dedifferentiation.

Additionally, the chondrogenic potential of chondrocyte dedifferentiation was observed to vary with different concentrations of TIIA, which indicated that the stimulatory effect was concentration-dependent. However, it should be noted that the proliferation and viability of chondrocytes in vitro were promoted by TIIA at a concentration of $100-200 \mu \mathrm{g} / \mathrm{mL}$, but inhibited by TIIA $400 \mu \mathrm{g} / \mathrm{mL}$. Adaptive concentration (100-200 $\mu \mathrm{g} / \mathrm{mL})$ of TIIA could accelerate proliferation and maintain the viability of chondrocytes, as evidenced by morphological comparisons and the increased number of live cells in the TIIA-treated groups compared to the control. As described above, $100-200 \mu \mathrm{g} / \mathrm{mL}$ TIIA significantly promoted the secretion of GAG in chondrocytes, which was the main component of cartilage ECM revealed by Alcian blue staining. The results indicated that TIIA could support the network of ECM, thus contributing to the proliferation and phenotype maintenance of chondrocytes. Furthermore, at low concentrations (100-200 $\mu \mathrm{g} / \mathrm{mL})$, TIIA up-regulated the 
gene expression of Col II and SOX6 while down-regulating the gene expression of $\mathrm{Col} I$ and $\mathrm{Col} X$ (Figure 7). Previous studies showed that TIIA inhibited cartilage degeneration through suppressing apoptosis and inflammatory cytokine production (38). However, few studies have focused on chondrocyte dedifferentiation and cartilage regeneration. The current study suggested that TIIA could prevent chondrocytes from dedifferentiation. Moreover, cell-loaded spheres pre-treated with TIIA had better cartilage repair in the rat model (Figure 9).

As an early marker of chondrogenesis, Col II contributes to $90 \%$ of total collagen content and forms the fibrils, which give cartilage its tensile strength. Furthermore, $\mathrm{Col}$ II can protect chondrocytes and the ECM in cartilage by reversing cartilage degradation (39). Chondrocyte hypertrophy represents a late and critical stage of cartilage development. Col X, a chondrocyte hypertrophy marker, is closely related to dedifferentiation, indicating that TIIA could regulate chondrogenesis by inhibiting chondrocyte hypertrophy. The SOX family of transcription factors are believed to be closely associated with chondrogenesis and development (40), among which SOX9 is considered to be the master regulator (41). SOX6 is another SOX transcription factor with a high degree of homology to SOX9 (42). Genetic research has indicated that SOX5 and SOX6 are also critical transcription factors for cartilage development, and are both transcriptional targets of SOX9 (43). Therefore, our results suggest that while the effect of TIIA was mediated through multiple regulatory mechanisms, SOX6 is likely to be a direct target which coordinates the chondrocyte dedifferentiation process.

\section{Conclusions}

In summary, a network pharmacology method was used to investigate the pharmacological effects of TIIA on chondrocyte dedifferentiation. TIIA might serve as a potential candidate to promote chondrocyte proliferation and phenotype maintenance in OA treatment. This study also provides a possible combination strategy for investigating the mechanisms of monomers of the TIIA.

\section{Acknowledgments}

We sincerely thank Dr. Yijie Zhang for his assistance in designing the study and experiments.

Funding: This work was supported by the National Natural Science Foundation of China (No. 31971272).

\section{Footnote}

Reporting Checklist: The authors have completed the ARRIVE reporting checklist. Available at http://dx.doi. org/10.21037/atm-20-4023

Peer Review File: Available at http://dx.doi.org/10.21037/ atm-20-4023

Conflicts of Interest: All authors have completed the ICMJE uniform disclosure form (available at http://dx.doi. org/10.21037/atm-20-4023). The authors have no conflicts of interest to declare.

Etbical Statement: The authors are accountable for all aspects of the work in ensuring that questions related to the accuracy or integrity of any part of the work are appropriately investigated and resolved. All animal experiments were approved by the Forth Military Medical University Animal Ethics Committee (permission number: FMMU20180712), and were implemented complying with the Guidelines for the Care and Use of Laboratory Animals.

Open Access Statement: This is an Open Access article distributed in accordance with the Creative Commons Attribution-NonCommercial-NoDerivs 4.0 International License (CC BY-NC-ND 4.0), which permits the noncommercial replication and distribution of the article with the strict proviso that no changes or edits are made and the original work is properly cited (including links to both the formal publication through the relevant DOI and the license). See: https://creativecommons.org/licenses/by-nc-nd/4.0/.

\section{References}

1. Griffin TM, Fermor B, Huebner JL, et al. Diet-induced obesity differentially regulates behavioral, biomechanical, and molecular risk factors for osteoarthritis in mice. Arthritis Res Ther 2010;12:R130.

2. Hunter DJ, Bierma-Zeinstra S. Osteoarthritis. Lancet 2019;393:1745-59.

3. Son YO, Kim HE, Choi WS, et al. RNA-binding protein ZFP36L1 regulates osteoarthritis by modulating members of the heat shock protein 70 family. Nat Commun 2019;10:77.

4. Argoff CE, Gloth FM. Topical nonsteroidal antiinflammatory drugs for management of osteoarthritis 
in long-term care patients. Ther Clin Risk Manag 2011;7:393-9.

5. Charlier E, Deroyer C, Ciregia F, et al. Chondrocyte dedifferentiation and osteoarthritis (OA). Biochem Pharmacol 2019;165:49-65.

6. Rosenzweig DH, Ou SJ, Quinn TM. P38 mitogenactivated protein kinase promotes dedifferentiation of primary articular chondrocytes in monolayer culture. J Cell Mol Med 2013;17:508-17.

7. Findlay DM, Atkins GJ. Osteoblast-chondrocyte interactions in osteoarthritis. Curr Osteoporos Rep 2014;12:127-34.

8. Xia H, Cao D, Yang F, et al. Jiawei Yanghe decoction ameliorates cartilage degradation in vitro and vivo via $W$ nt/beta-catenin signaling pathway. Biomed Pharmacother 2020;122:109708.

9. Ziadlou R, Barbero A, Stoddart MJ, et al. Regulation of inflammatory response in human osteoarthritic chondrocytes by novel herbal small molecules. Int J Mol Sci 2019;20:5745.

10. Bai B, Li Y. Danshen prevents articular cartilage degeneration via antioxidation in rabbits with osteoarthritis. Osteoarthritis Cartilage 2016;24:514-20.

11. Chen Z, Xu H. Anti-inflammatory and immunomodulatory mechanism of tanshinone IIA for atherosclerosis. Evid Based Complement Alternat Med 2014;2014:267976.

12. Li YI, Elmer G, Leboeuf RC. Tanshinone IIA reduces macrophage death induced by hydrogen peroxide by upregulating glutathione peroxidase. Life Sci 2008;83:557-62.

13. Zhang Y, Jiang P, Ye M, et al. Tanshinones: sources, pharmacokinetics and anti-cancer activities. Int J Mol Sci 2012;13:13621-66.

14. Fang J, Little PJ, Xu S. Atheroprotective effects and molecular targets of tanshinones derived from herbal medicine Danshen. Med Res Rev 2018;38:201-28.

15. Lee S. Systems biology - a pivotal research methodology for understanding the Mechanisms of Traditional Medicine. J Pharmacopuncture 2015;18:11-8.

16. Shi MJ, Yan XL, Dong BS, et al. A network pharmacology approach to investigating the mechanism of Tanshinone IIA for the treatment of liver fibrosis. J Ethnopharmacol 2020;253:112689.

17. Liu X, Ouyang S, Yu B, et al. PharmMapper server: a web server for potential drug target identification using pharmacophore mapping approach. Nucleic Acids Res 2010;38:W609-14.

18. Daina A, Michielin O, Zoete V. Swiss'TargetPrediction: updated data and new features for efficient prediction of protein targets of small molecules. Nucleic Acids Res 2019;47:W357-64.

19. Wishart DS, Knox C, Guo AC, et al. DrugBank: a comprehensive resource for in silico drug discovery and exploration. Nucleic Acids Res 2006;34:D668-72.

20. Ru J, Li P, Wang J, et al. TCMSP: a database of systems pharmacology for drug discovery from herbal medicines. J Cheminform 2014;6:13.

21. Liu Z, Guo F, Wang Y, et al. BATMAN-TCM: a Bioinformatics Analysis Tool for Molecular mechANism of Traditional Chinese Medicine. Sci Rep 2016;6:21146.

22. Chen CY. TCM Database@Taiwan: the world's largest traditional Chinese medicine database for drug screening in silico. PLoS One 2011;6:e15939.

23. Mandloi S, Chakrabarti S. PALM-IST: Pathway Assembly from Literature Mining--an Information Search Tool. Sci Rep 2015;5:10021.

24. Pathan M, Keerthikumar S, Ang CS, et al. FunRich: An open access standalone functional enrichment and interaction network analysis tool. Proteomics 2015;15:2597-601.

25. Zhang YS, Zhang PH, Liu XC, et al. Effects of Tanshinon IIA on dedifferentiation of chondrocytes from rat knee. Chinese Journal of Bone and Joint Injury 2017;32:942-5.

26. Mata-Miranda MM, Martinez-Martinez CM, NoriegaGonzalez JE, et al. Morphological, genetic and phenotypic comparison between human articular chondrocytes and cultured chondrocytes. Histochem Cell Biol 2016;146:183-9.

27. Schulze-Tanzil G. Activation and dedifferentiation of chondrocytes: implications in cartilage injury and repair. Ann Anat 2009;191:325-38.

28. Minegishi Y, Hosokawa K, Tsumaki N. Time-lapse observation of the dedifferentiation process in mouse chondrocytes using chondrocyte-specific reporters. Osteoarthritis Cartilage 2013;21:1968-75.

29. Zhang R, Zhu X, Bai H, et al. Network pharmacology databases for Traditional Chinese Medicine: review and assessment. Front Pharmacol 2019;10:123.

30. Zhang W, Huai Y, Miao Z, et al. Systems pharmacology for investigation of the mechanisms of action of Traditional Chinese Medicine in drug discovery. Front Pharmacol 2019;10:743.

31. Jia Q, Zhu R, Tian Y, et al. Salvia miltiorrhiza in diabetes: a review of its pharmacology, phytochemistry, and safety. Phytomedicine 2019;58:152871.

32. Shi MJ, Dong BS, Yang WN, et al. Preventive and 
therapeutic role of Tanshinone A in hepatology. Biomed Pharmacother 2019;112:108676.

33. Ding $M, M a W$, Wang $X$, et al. A network pharmacology integrated pharmacokinetics strategy for uncovering pharmacological mechanism of compounds absorbed into the blood of Dan-Lou tablet on coronary heart disease. J Ethnopharmacol 2019;242:112055.

34. Zhang GX, Zhang YY, Zhang XX, et al. Different network pharmacology mechanisms of Danshen-based Fangjis in the treatment of stable angina. Acta Pharmacol Sin 2018;39:952-60.

35. Wu R, Dong S, Cai FF, et al. Active compounds derived from Fuzheng Huayu formula protect hepatic parenchymal cells from apoptosis based on network pharmacology and transcriptomic analysis. Molecules 2019;24:338.

36. Luan L, Liang Z. Tanshinone IIA protects murine chondrogenic ATDC5 cells from lipopolysaccharideinduced inflammatory injury by down-regulating microRNA-203a. Biomed Pharmacother 2018;103:628-36.

37. Jia PT, Zhang XL, Zuo HN, et al. Articular cartilage degradation is prevented by tanshinone IIA through inhibiting apoptosis and the expression of inflammatory

Cite this article as: Zhang Y, Sun L, Liu X, Zhu D, Dang J, Xue Y, Fan H. Investigating the protective effect of tanshinone IIA against chondrocyte dedifferentiation: a combined molecular biology and network pharmacology approach. Ann Transl Med 2021;9(3):249. doi: 10.21037/atm-20-4023 cytokines. Mol Med Rep 2017;16:6285-9.

38. Wang JG, Bondy SC, Zhou L, et al. Protective effect of Tanshinone IIA against infarct size and increased HMGB1, NFkappaB, GFAP and apoptosis consequent to transient middle cerebral artery occlusion. Neurochem Res 2014;39:295-304.

39. Yan B, Zhou L, Wang C, et al. Intra-Articular Injection of Fructus Ligustri Lucidi Extract Attenuates Pain Behavior and Cartilage Degeneration in Mono-Iodoacetate Induced Osteoarthritic Rats. Front Pharmacol 2018;9:1360.

40. Akiyama H, Lefebvre V. Unraveling the transcriptional regulatory machinery in chondrogenesis. J Bone Miner Metab 2011;29:390-5.

41. Akiyama H. Control of chondrogenesis by the transcription factor Sox9. Mod Rheumatol 2008;18:213-9.

42. Ikeda T, Kawaguchi H, Kamekura S, et al. Distinct roles of Sox 5, Sox6, and Sox9 in different stages of chondrogenic differentiation. J Bone Miner Metab 2005;23:337-40.

43. Smits P, Dy P, Mitra S, et al. Sox 5 and Sox6 are needed to develop and maintain source, columnar, and hypertrophic chondrocytes in the cartilage growth plate. J Cell Biol 2004;164:747-58. 